\title{
Exotic vs. conventional scaling and universality in a disordered bilayer quantum Heisenberg antiferromagnet
}

\author{
Rastko Sknepnek, ${ }^{1}$ Thomas Vojta, ${ }^{1}$ and Matthias Vojta ${ }^{2}$ \\ ${ }^{1}$ Physics Department, University of Missouri - Rolla, Rolla, MO 65409 \\ ${ }^{2}$ Institut für Theorie der Kondensierten Materie, Universität Karlsruhe, 76128 Karlsruhe, Germany
}

(Dated: December 4, 2018)

\begin{abstract}
We present Monte-Carlo simulations of a two-dimensional (2d) bilayer quantum Heisenberg antiferromagnet with random dimer dilution. In contrast to exotic scaling scenarios found in other random quantum systems, the quantum phase transition in this system is characterized by a finitedisorder fixed point with power-law scaling. After accounting for corrections to scaling, with a leading irrelevant exponent of $\omega \approx 0.48$, we find universal critical exponents $z=1.310(6)$ and $\nu=1.16(3)$. We discuss the consequences of these findings and suggest new experiments.
\end{abstract}

Quantum phase transitions (QPT) under the influence of quenched disorder are a topic of great current interest. Experimental examples range from localized [1] and itinerant 2] quantum magnets to heavy-fermion compounds 3], high-temperature superconductors [4], and to metalinsulator [5] and superconductor-insulator transitions [6]. These systems display rich new physics but many are still poorly understood. In the context of classical phase transitions, the interplay between disorder and critical fluctuations has a long history. Harris 7] derived a criterion for the stability of a critical point against disorder: If the correlation length exponent $\nu$ fulfills the inequality $\nu>2 / d$, where $d$ is the spatial dimensionality, the critical behavior is not influenced by weak disorder. If a clean critical point violates the Harris criterion, the generic result of introducing disorder is a new (finitedisorder) critical point with power-law scaling and new critical exponents which fulfill the Harris criterion [8].

At QPTs, order-parameter fluctuations in space and time must be considered. Quenched disorder is perfectly correlated in time direction. As a result, disorder effects at QPTs are generically stronger than at classical transitions. Prominent consequences are the infiniterandomness critical points in $1 \mathrm{~d}$ random spin chains [9] and in $1 \mathrm{~d}[10$ and $2 \mathrm{~d}[11,12]$ random quantum Ising models. At these critical points, the dynamical scaling is activated, i.e., correlation time $\xi_{\tau}$ and correlation length $\xi$ obey $\ln \xi_{\tau} \sim \xi^{\mu}$. (At conventional critical points, this relation is a power law, $\xi_{\tau} \sim \xi^{z}$, with a universal dynamical exponent $z$ ). In itinerant electron systems, the effects of impurities can be even more dramatic. For Ising symmetry, the interplay of quenched disorder and Landau damping of the order parameter fluctuations completely destroys the sharp QPT by smearing 13]. Further exotic phenomena include non-universal, continuously varying exponents, observed in the Griffiths region associated with a QPT [10, 11, 14] or at certain impurity QPTs [15]. On the other hand, the stable low-energy (as opposed to critical) fixed point of random Heisenberg models in $d \geq 2$ has been shown to be conventional [16]. Preliminary results [12] for the critical point in these models suggest that the infinite-randomness fixed point is unstable, but no definite conclusion on the fate of the transition has been reached. These results lead to the general question whether all QPTs in presence of quenched disorder are unconventional.

In this Letter, we provide a "proof of principle" that this is not the case: The QPT of a dimer-diluted spin- $1 / 2$ bilayer quantum Heisenberg antiferromagnet is shown to exhibit a conventional finite-disorder critical point with power-law dynamical scaling and universal critical exponents. After accounting for corrections to scaling characterized by an irrelevant exponent $\omega \approx 0.48$ we find the asymptotic dynamical and correlation length exponents to be $z=1.310(6)$ and $\nu=1.16(3)$ (fulfilling the Harris criterion $\nu>2 / d=1[$ 7, $[8]$ ).

Our starting point is a bilayer quantum Heisenberg antiferromagnet as depicted in the inset of Fig. [1 The spins in each $2 \mathrm{~d}$ layer interact via nearest neighbor exchange $J_{\|}$, and the interplane coupling is $J_{\perp}$. The clean version of this model has been studied extensively [17, 18]. For $J_{\perp} \gg J_{\|}$, neighboring spins from the two layers form singlets, and the ground state is paramagnetic. In contrast, for $J_{\|} \gg J_{\perp}$ the system develops Néel order. Both phases are separated by a QPT at $J_{\perp} / J_{\|} \approx 2.525$. Random disorder is introduced by removing pairs (dimers) of adjacent spins, one from each layer. The Hamiltonian of the model with dimer dilution is:

$$
H=J_{\|} \sum_{\substack{\langle i, j\rangle \\ a=1,2}} \epsilon_{i} \epsilon_{j} \hat{\mathbf{S}}_{i, a} \cdot \hat{\mathbf{S}}_{j, a}+J_{\perp} \sum_{i} \epsilon_{i} \hat{\mathbf{S}}_{i, 1} \cdot \hat{\mathbf{S}}_{i, 2},
$$

and $\epsilon_{i}=0\left(\epsilon_{i}=1\right)$ with probability $p(1-p)$.

The phase diagram of the dimer-diluted bilayer Heisenberg model has been studied by Sandvik [19] and Vajk and Greven 20], see Fig. [1 For small $J_{\perp}$, magnetic order survives up to the percolation threshold $p_{p} \approx 0.4072$, and a multicritical point exists at $p=p_{p}$ and $J_{\perp} / J_{\|} \approx 0.16$. We focus on the generic transition at $0<p<p_{p}$, driven by $J_{\perp}$, where the results of Refs. [19, 20] are inconclusive.

To determine the critical behavior at the QPT effectively, we proceed by mapping the quantum Hamiltonian 


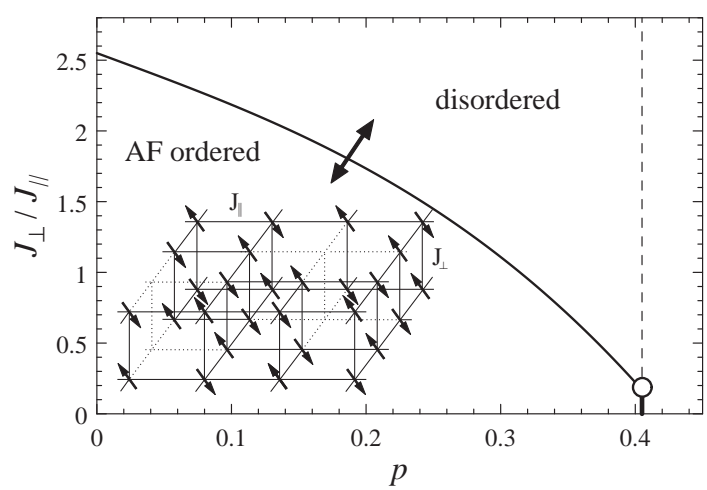

FIG. 1: Phase diagram 20] of the diluted bilayer Heisenberg antiferromagnet, as function of $J_{\perp} / J_{\|}$and dilution $p$. The dashed line is the percolation threshold, the open dot is the multicritical point of Refs. [19, 20]. The arrow indicates the QPT studied here. Inset: The model: Quantum spins (arrows) reside on the two parallel square lattices. The spins in each plane interact with the coupling strength $J_{\|}$. Interplane coupling is $J_{\perp}$. Dilution is done by removing dimers.

(11) onto a classical model. First we note that the lowenergy properties of bilayer quantum antiferromagnets are represented by a $(2+1)$-dimensional $\mathrm{O}(3)$ quantum rotor model [21] with the rotor coordinate $\hat{\mathbf{n}}_{i}$ corresponding to $\hat{\mathbf{S}}_{i, 1}-\hat{\mathbf{S}}_{i, 2}$ and the angular momentum $\hat{\mathbf{L}}_{i}$ representing $\hat{\mathbf{S}}_{i, 1}+\hat{\mathbf{S}}_{i, 2}$ (see, e.g., chapter 5 of Ref. [22]). This quantum rotor model in turn is equivalent to a $3 \mathrm{~d}$ classical Heisenberg model with the disorder perfectly correlated in imaginary time direction, as can be easily seen from a path integral representation of the partition function. Thus, our classical Hamiltonian reads:

$$
H=K \sum_{\langle i, j\rangle, \tau} \epsilon_{i} \epsilon_{j} \mathbf{n}_{i, \tau} \cdot \mathbf{n}_{j, \tau}+K \sum_{i, \tau} \epsilon_{i} \mathbf{n}_{i, \tau} \cdot \mathbf{n}_{i, \tau+1},
$$

where $\mathbf{n}_{i, \tau}$ is an $\mathrm{O}(3)$ unit vector. The coupling constant $\beta K$ of the classical model is related to the ratio $J_{\|} / J_{\perp}$ of the quantum model. Here, $\beta \equiv 1 / T$ where $T$ is an effective "classical" temperature, not equal to the real temperature which is zero. We set $K=1$ and drive the classical system through the transition by tuning the classical temperature $T$.

As an aside, we note that dimer dilution in the quantum model (11) does not introduce random Berry phases because the Berry phase contributions from the two spins of each unit cell cancel 21, 22]. In contrast, for site dilution, the physics changes completely: The random Berry phases (which have no classical analogue) are equivalent to impurity-induced moments [23], and those become weakly coupled via bulk excitations. Thus, for all $p<p_{p}$ the ground state shows long-range order, independent of $J_{\perp} / J_{\|}$! This effect is absent for dimer dilution, and both phases of the clean system survive for small $p$ 24].

The classical model (2) is studied by Monte-Carlo simulations using the efficient Wolff cluster algorithm
[25, 26]. We investigate linear sizes up to $L=120$ in space direction and $L_{\tau}=384$ in imaginary time, for impurity concentrations $p=\frac{1}{8}, \frac{1}{5}, \frac{2}{7}$ and $\frac{1}{3}$. The results are averaged over $10^{3}-10^{4}$ disorder realizations. Each sample is equilibrated using 100 Monte-Carlo sweeps (spinflips per site). For large dilutions, $p=\frac{2}{7}$ and $\frac{1}{3}$ we perform both Wolff and Metropolis sweeps to equilibrate small dangling clusters. During the measurement period of another 100-200 sweeps we calculate magnetization, susceptibility, specific heat and correlation functions.

A quantity particularly suitable to locate the critical point and to extract high precision values for the exponents $z$ and $\nu$ is the Binder ratio:

$$
g_{a v}=\left[1-\frac{\left\langle|\mathbf{M}|^{4}\right\rangle}{3\left\langle|\mathbf{M}|^{2}\right\rangle^{2}}\right]_{a v},
$$

where $\mathbf{M}=\sum_{i, \tau} \mathbf{n}_{i . \tau},[\ldots]_{a v}$ denotes the disorder average and $\langle\ldots\rangle$ denotes the Monte-Carlo average for each sample. This quantity has scale dimension 0 . Thus, its finite-size scaling form is given by

$$
\begin{aligned}
& g_{a v}=\tilde{g}_{C}\left(t L^{1 / \nu}, L_{\tau} / L^{z}\right) \quad \text { or } \\
& g_{a v}=\tilde{g}_{A}\left(t L^{1 / \nu}, \log \left(L_{\tau}\right) / L^{\mu}\right)
\end{aligned}
$$

for conventional scaling or for activated scaling, respectively. Two important characteristics follow: (i) For fixed $L, g_{a v}$ has a peak as a function of $L_{\tau}$. The peak position $L_{\tau}^{\max }$ marks the optimal sample shape, where the ratio $L_{\tau} / L$ roughly behaves like the corresponding ratio of the correlation lengths in time and space directions, $\xi_{\tau} / \xi$. At the critical temperature $T_{c}$, the peak value $g_{a v}^{\max }$ is independent of $L$. Thus, for power law scaling, plotting $g_{a v}$ vs. $L_{\tau} / L_{\tau}^{\max }$ at $T_{c}$ should collapse the data, without the need for a value of $z$. In contrast, for activated scaling the $g_{a v}$ data should collapse when plotted as a function of $\log \left(L_{\tau}\right) / \log \left(L_{\tau}^{\max }\right)$. (ii) For samples of the optimal shape $\left(L_{\tau}=L_{\tau}^{\max }\right)$, plots of $g_{a v}$ vs. temperature for different $L$ cross at $T_{c}$. Based on these two characteristics, we use a simple iterative procedure to determine both the optimal shapes and the location of the critical point.

We now turn to our results. To distinguish between activated and power-law dynamical scaling we perform a series of calculations at the critical temperature. The upper panel of Fig. 2] shows the Binder ratio $g_{a v}$ as a function of $L_{\tau}$ for various $L=5 \ldots 100$ and dilution $p=\frac{1}{5}$ at $T=T_{c}=1.1955$. The statistical error of $g_{a v}$ is below $0.1 \%$ for the smaller sizes and not more than $0.2 \%$ for the largest systems. As expected at $T_{c}$, the maximum Binder ratio for each of the curves does not depend on $L$. To test the conventional power-law scaling form, eq. (4), we plot $g_{a v} / g_{a v}^{\max }$ as a function of $L_{\tau} / L_{\tau}^{\max }$ in the lower panel of Fig. 2] The data scale extremely well, giving statistical errors of $L_{\tau}^{\max }$ in the range between $0.3 \%$ and $1 \%$. For comparison, the inset shows a plot of $g_{a v}$ as a function of $\log \left(L_{\tau}\right) / \log \left(L_{\tau}^{\max }\right)$ corresponding to eq. (5). The data clearly do not scale which rules out the activated scaling scenario. The results for the other impurity 


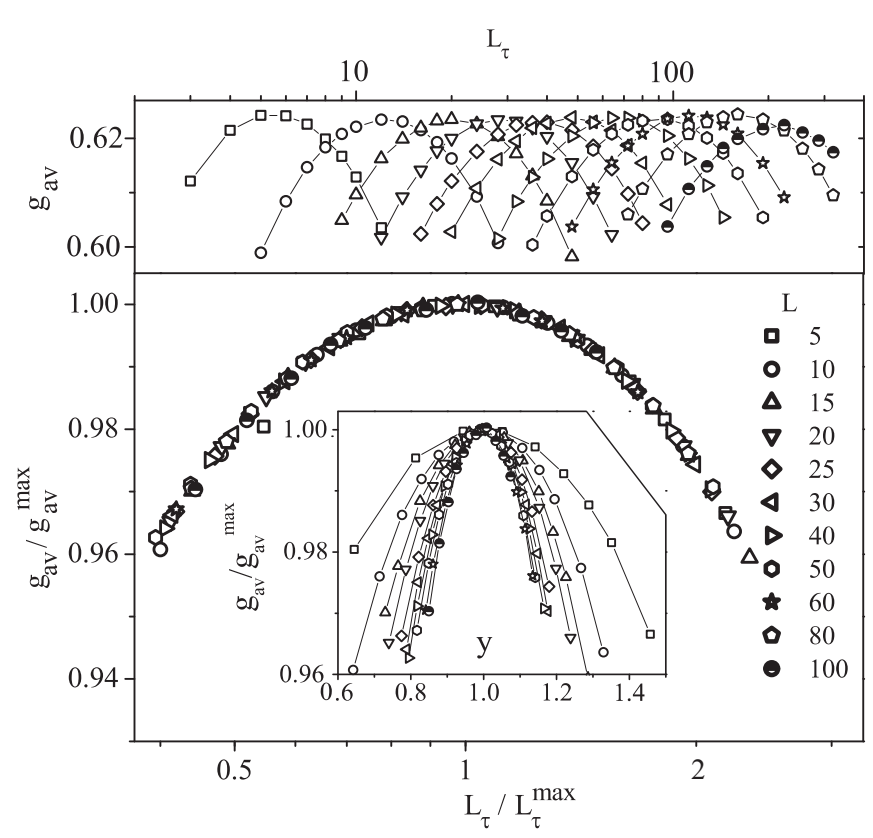

FIG. 2: Upper panel: Binder ratio $g_{a v}$ as a function of $L_{\tau}$ for various $L\left(p=\frac{1}{5}\right)$. Lower panel: Power-law scaling plot $g_{a v} / g_{a v}^{\max }$ vs. $L_{\tau} / L_{\tau}^{\max }$ Inset: Activated scaling plot $g_{a v} / g_{a v}^{\max }$ vs. $y=\log \left(L_{\tau}\right) / \log \left(L_{\tau}^{\max }\right)$.

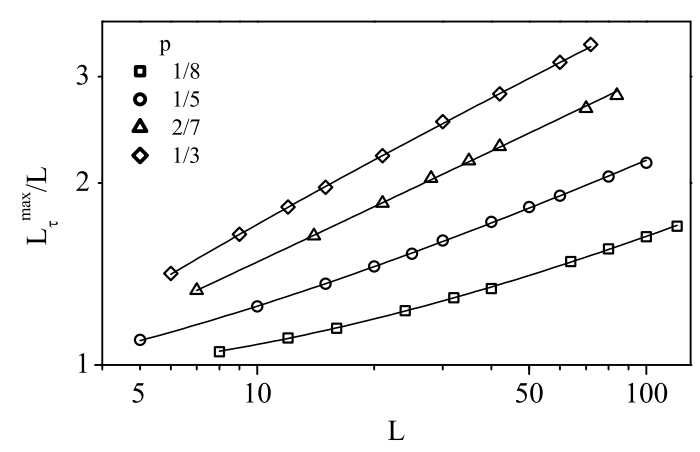

FIG. 3: $L_{\tau}^{\max } / L$ vs. $L$ for four disorder concentrations $p=\frac{1}{8}$, $\frac{1}{5}, \frac{2}{7}$ and $\frac{1}{3}$. Solid lines: Fit to $L_{\tau}^{\max }=a L^{z}\left(1+b L^{-\omega_{1}}\right)$ with $z=1.310(6)$ and $\omega_{1}=0.48(3)$.

concentrations $p=\frac{1}{8}, \frac{2}{7}, \frac{1}{3}$ are completely analogous.

Having established conventional power-law dynamical scaling, we proceed to determine the dynamical exponent $z$. In Fig. 3] we plot $L_{\tau}^{\max }$ vs. $L$ for all four dilutions $p$. The curves show significant deviations from pure powerlaw behavior which can be attributed to corrections to scaling due to irrelevant operators. In such a situation, a direct power-law fit of the data will only yield effective exponents. To find the true asymptotic exponents we take the leading correction to scaling into account by using the ansatz $L_{\tau}^{\max }(L)=a L^{z}\left(1+b L^{-\omega_{1}}\right)$ with universal (dilution-independent) exponents $z$ and $\omega_{1}$ but dilutiondependent $a$ and $b$. A combined fit of all four curves gives $z=1.310(6)$ and $\omega_{1}=0.48(3)$ where the number in brackets is the standard deviation of the last given digit.

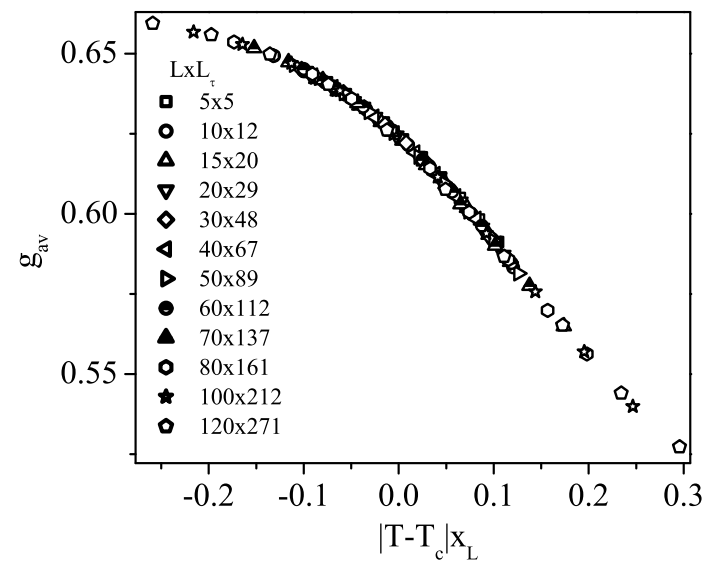

FIG. 4: Scaling plot of $g_{a v}$ vs. $\left(T-T_{c}\right) x_{L}$ for $p=0.2 . x_{L}$ is the factor necessary to scale the data onto a master curve.

The fit is of high quality $\left(\chi^{2} \approx 0.7\right)$ and robust against removing complete data sets or removing points form the lower or upper end of each set. We thus conclude that the asymptotic dynamical exponent $z$ is indeed universal. (Note that the leading corrections to scaling vanish very close to $p=\frac{2}{7}$; the curvature of the $L_{\tau}^{\max }(L)$ curves in Fig. 3 is opposite above and below this concentration.)

To find the correlation length exponent $\nu$, we perform simulations in the vicinity of $T_{c}$ for samples with the optimal shape $\left(L_{\tau}=L_{\tau}^{\max }\right)$ to keep the second argument of the scaling function (4) constant. Fig. (4 shows a scaling plot of $g_{a v}$ vs. $T$ for impurity concentration $p=\frac{1}{5}$. Again, the data scale very well, but since the scaling function lacks the characteristic maximum, the error of the resulting scaling factor $x_{L}$ is somewhat larger $(1 \ldots 2 \%)$ than that of $L_{\tau}^{\max }$. The same quality of scaling was achieved for the other dilutions. Fig. [5] shows the scaling factor $x_{L}$ vs. $L$ for all four data sets. A combined fit to the ansatz $x_{L}=c L^{1 / \nu}\left(1+d L^{-\omega_{2}}\right)$ where $\nu$ and $\omega_{2}$ are universal, gives $\nu=1.16(3)$ and $\omega_{2}=0.5(1)$. As above, the fit is robust and of high quality $\left(\chi^{2} \approx 1.2\right)$. Importantly, as expected for the true asymptotic exponent, $\nu$ fulfills the Harris criterion [7],$\nu>2 / d=1$. Note that both irrelevant exponents $\omega_{1}$ and $\omega_{2}$ agree within their error bars, suggesting that the same irrelevant operator controls the leading corrections to scaling for both $z$ and $\nu$.

We have also calculated total magnetization and susceptibility. The corresponding exponents $\beta / \nu=0.56(5)$ and $\gamma / \nu=2.15(10)$ have slightly larger error bars than $z$ and $\nu$. Nonetheless, they fulfill the hyperscaling relation $2 \beta+\gamma=(d+z) \nu$ which is another argument for our results being asymptotic rather than effective exponents.

In summary, we have performed Monte-Carlo simulations of a 3d classical Heisenberg model with linear impurities which is in the same universality class as the dimer-diluted bilayer quantum Heisenberg antiferromagnet. We have shown that the QPT in this system is controlled by a conventional, finite-disorder critical point 


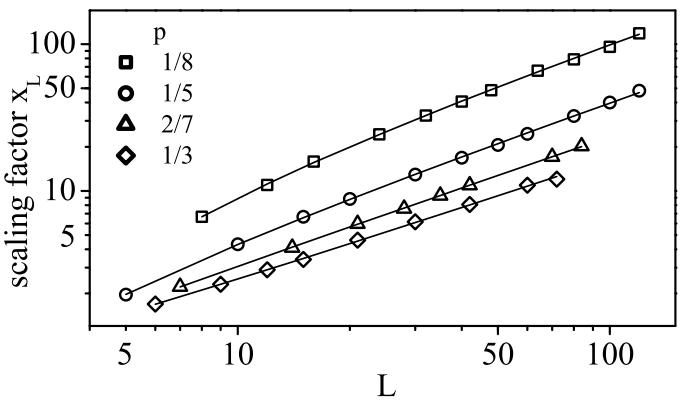

FIG. 5: Scaling factor vs. $L$ for four disorder concentrations $p=\frac{1}{8}, \frac{1}{5}, \frac{2}{7}$ and $\frac{1}{3}$. Solid lines: Fit to $x_{L}=c L^{1 / \nu}\left(1+d L^{-\omega_{2}}\right)$ with $\nu=1.16(3)$ and $\omega_{2}=0.5(1)$.

with power-law dynamical scaling and universal exponents. (Note that the Ising version of our model, the diluted $2 \mathrm{~d}$ random transverse Ising model, shows an infinite-randomness critical point [11, 28].)

Let us compare our results to previous work. The multicritical point at $p=p_{p}$ and $J_{\perp} / J_{\|} \approx 0.16$, found in Refs. [19, 20], has a dynamical exponent $z \approx 1.3$. Within the error bars, this value coincides with the one found here for the generic $p<p_{p}$ transition. We see no a-priori reason for this coincidence, so far it is unclear whether or not it is accidental. Vajk and Greven [20] also quote exponents for $p<p_{c}$. At dilution $p=0.25$ they find $z=1.07$ and $\nu=0.89$, different from our results. However, as the authors of Ref. 20] point out, a value of $\nu<1$ violates the Harris criterion, indicating that it represents an effective rather than an asymptotic exponent. It would also be useful to compare our findings with analytical results. To the best of our knowledge, the only quantitative result is a resummation of the 2loop $\epsilon$-expansion [27]. The predicted exponents significantly differ from ours; but they also violate the Harris criterion, casting doubt on their validity.

Finally, we comment on experiments. If chemical doping replaces magnetic by non-magnetic ions in an antiferromagnet, e.g., $\mathrm{Cu}$ by $\mathrm{Zn}$ in $\mathrm{YBa}_{2} \mathrm{Cu}_{3} \mathrm{O}_{6}$, the case of site rather than dimer dilution is realized. The most promising way to achieve bond dilution is the introduction of strong antiferromagnetic intra-dimer bonds at random locations. Thus we propose to study magnetic transitions in bond-disordered systems; those transitions can be expected to be in the same universality class as the one studied here. One candidate material - albeit $3 \mathrm{~d}-$ is $(\mathrm{Tl}, \mathrm{K}) \mathrm{CuCl}_{3}$ [29] under pressure; interesting quasi-2d compounds are $\mathrm{SrCu}_{2}\left(\mathrm{BO}_{3}\right)_{2}$ or $\mathrm{BaCuSi}_{2} \mathrm{O}_{6}$, where suitable dopants remain to be found.

We acknowledge partial support from the University of Missouri Research Board, from the NSF under grant No.
DMR-0339147 and from the DFG Center for Functional Nanostructures Karlsruhe.

[1] W. Wu et al., Phys. Rev. Lett. 67, 2076 (1991).

[2] J. DiTusa et al., cond-mat/0306541

[3] C. L. Seaman et al., Phys. Rev. Lett. 67, 2882 (1991); M. C. de Andrade et al., ibid. 81, 5620 (1998).

[4] C. Panagopoulos et al., Phys. Rev. B 66, 064501 (2002).

[5] S. V. Kravchenko et al., Phys. Rev. B 517038 (1995); E. Abrahams, S. Kravchenko, and M. Sarachik, Rev. Mod. Phys. 73, 251 (2001).

[6] A. F. Hebard and M. A. Paalanen, Phys. Rev. Lett. 65, 927 (1990).

[7] A. B. Harris, J. Phys. C 7, 1671 (1974).

[8] J. Chayes et al., Phys. Rev. Lett. 57, 2999 (1986).

[9] S. K. Ma, C. Dasgupta, and C.-K. Hu, Phys. Rev. Lett. 43, 1434 (1979); R. N. Bhatt and P. A. Lee, ibid. 48, 344 (1982); D. S. Fisher, Phys. Rev. B 50, 3799 (1994).

[10] D. S. Fisher, Phys. Rev. Lett. 69, 534 (1992); Phys. Rev. B 51, 6411 (1995); A. P. Young and H. Rieger, ibid. 53, 8486 (1996).

[11] C. Pich et al., Phys. Rev. Lett. 81, 5916 (1998);

[12] O. Motrunich et al., Phys. Rev. B 61, 1160 (2000).

[13] T. Vojta, Phys. Rev. Lett. 90, 107202 (2003).

[14] R. B. Griffiths, Phys. Rev. Lett. 23, 17 (1969); B. M. McCoy, Phys. Rev. Lett. 23, 383 (1969).

[15] A. Georges and A. M. Sengupta, Phys. Rev. Lett. 74, 2808 (1995), and references therein.

[16] Y.-C. Lin et al., Phys. Rev. B 68, 024424 (2003).

[17] K. Hida, J. Phys. Soc. Jpn. 59, 2230 (1990); A. J. Millis and H. Monien, Phys. Rev. Lett. 70, 2810 (1993).

[18] A. W. Sandvik and D. J. Scalapino, Phys. Rev. Lett. 72, 2777 (1994); P. V. Shevchenko, A. W. Sandvik, and O. P. Sushkov, Phys. Rev. B 61, 3475 (2000).

[19] A. W. Sandvik, Phys. Rev. Lett. 89, 177201 (2002).

[20] O. P. Vajk and M. Greven, Phys. Rev. Lett. 89, 177202 (2002).

[21] S. Chakravarty, B. I. Halperin and D. R. Nelson, Phys. Rev. B 39, 2344 (1989).

[22] S. Sachdev, Quantum Phase Transitions, Cambridge University Press, Cambridge (1999).

[23] S. Sachdev and M. Vojta, in Proceedings of the XIII International Congress on Mathematical Physics, eds. A. Fokas et al., International Press, Boston (2001).

[24] While random Berry phases can produce exotic behavior, they are not responsible for the infinite-randomness critical points or the strong quantum Griffiths singularities found in random quantum Ising models [10, 11].

[25] U. Wolff, Phys. Rev. Lett. 62, 361 (1989).

[26] Since the classical model is not frustrated, we can use cluster algorithms to reduce critical slowing down.

[27] V. Blavats'ka et al., Phys. Rev. B 67, 094404 (2003).

[28] T. Senthil and S. Sachdev, Phys. Rev. Lett. 77, 5292 (1996).

[29] A. Oosawa and H. Tanaka, Phys. Rev. B 65, 184437 (2002). 